\title{
Note on Askey-Wilson $q$-Contour Integral Formula
}

\author{
Jian-Ping Fang ${ }^{1}$ \\ ${ }^{1}$ School of Mathematical Science, Huaiyin Normal University, Huaian, Jiangsu, P. R. China \\ Correspondence: Jian-Ping Fang, School of Mathematical Science, Huaiyin Normal University, Huaian 223300, \\ Jiangsu, P. R. China. E-mail: fjp7402@163.com
}

Received: February 11, 2014 Accepted: March 13, 2014 Online Published: April 18, 2014

doi:10.5539/jmr.v6n2p59 URL: http://dx.doi.org/10.5539/jmr.v6n2p59

\begin{abstract}
We use Askey-Wilson $q$-contour integral formula and the $q$-Saalschütz's summation to derive a new recurring $q$ contour integral formula in this paper. Using this formula, we present a simple proof of the Sears' transformation of terminating balanced ${ }_{4} \Phi_{3}$ series.
\end{abstract}

Keywords: $q$-contour integral, Askey and Wilson $q$-contour integral, $q$-Saalschütz's summation formula, Sears' terminating ${ }_{4} \Phi_{3}$ transformation formula

\section{Introduction and Main Result}

We adopt the notations used by Gasper and Rahman (2004). Throughout this paper, it is supposed that $0<|q|<1$. For any complex parameter $a$, the $q$-shifted factorial are defined as

$$
(a ; q)_{0}=1,(a ; q)_{n}=\prod_{k=0}^{n-1}\left(1-a q^{k}\right),(a ; q)_{\infty}=\prod_{k=0}^{\infty}\left(1-a q^{k}\right), n=1,2, \cdots
$$

The following compact notation for the multiple $q$-shifted factorial is used:

$$
\left(a_{1}, a_{2}, \cdots, a_{m} ; q\right)_{n}=\left(a_{1} ; q\right)_{n}\left(a_{2} ; q\right)_{n} \cdots\left(a_{m} ; q\right)_{n}, m, n=0,1,2, \cdots .
$$

The basic hypergeometric series ${ }_{s} \Phi_{t}$ is given by:

$$
{ }_{s} \Phi_{t}\left(\begin{array}{cccc}
a_{1}, & a_{2}, & \cdots, & a_{s} \\
b_{1}, & b_{2}, & \cdots, & b_{t}
\end{array} ; q, \quad x\right)=\sum_{k=0}^{\infty} \frac{\left(a_{1}, a_{2} \cdots, a_{s} ; q\right)_{k}}{\left(q, b_{1}, \cdots, b_{t} ; q\right)_{k}}\left[(-1)^{k} q^{\left(\begin{array}{l}
k \\
2
\end{array}\right)}\right]^{1+t-s} x^{k},
$$

where $s, t=0,1,2, \cdots$.

For any complex numbers $a$ and $b$, the polynomial $P_{n}(a ; b)$ is defined as:

$$
P_{0}(a ; b)=1, P_{n}(a ; b)=(a-b)(a-b q) \cdots\left(a-b q^{n-1}\right), n \geq 1 .
$$

For $a \neq 0$, we define

$$
F_{n}(a ; b)=P_{n}(a ; b) P_{n}(1 / a ; b) .
$$

Since $q$-integral was introduced by Jackson (1910), it has been studied by many researchers who produced numerous literatures about it (see Andrews \& Askey, 1981; Askey, 1981; Askey \& Wilson, 1985; Costas-Santos \& Sánchez-Lara, 2013; Ismail \& Masson, 1994, 1995; Jackson, 1910; Liu, 2003; Wang, 2010). Inspired by Wang (2010), a new of $q$-contour integral formula have been derived in Fang (2014) from the following elegant AskeyWilson integral formula (consult Askey \& Wilson, 1985, Theorem 2.1)

$$
\frac{1}{2 \pi i} \int_{C} \frac{\left(z^{2}, z^{-2} ; q\right)_{\infty}}{(a z, a / z, b z, b / z, c z, c / z, d z, d / z ; q)_{\infty}} \frac{d z}{z}=\frac{2(a b c d ; q)_{\infty}}{(q, a b, a c, a d, b c, b d, c d ; q)_{\infty}},
$$

where the contour $C$ is a deformation of unit circle so that the poles of $1 /(a z, b z, c z, d z ; q)_{\infty}$ lie outside the contour and the origin and poles of $1 /(a / z, b / z, c / z, d / z ; q)_{\infty}$ lie inside the contour. 
In this paper, we continue to use this method to give a new recurring $q$-contour integral formula. Using this new recurring formula and the symmetry of the function, we present a simple proof of the famous Sears' transformation of terminating balanced ${ }_{4} \Phi_{3}$ series. And we also present two extension transformation formulas of terminating $\Phi_{3}$ series which are different from the extension presented in Fang (2007). The main result of this paper is stated as follows:

Theorem 1 Assume the pairwise products of $\{a, b, c, d\}$ as a multiset (i.e. both $a^{2}$ and ab are considered among the products) do not belong to the set of $\left\{q^{i}, i=0,-1,-2, \cdots\right\}$, and assume that there are no zero factors in the denominator, then

$$
\begin{aligned}
& \int_{C} \frac{\left(z^{2}, z^{-2} ; q\right)_{\infty}}{(a z, a / z, b z, b / z, c z, c / z, d z, d / z ; q)_{\infty}} \prod_{j=1}^{m+1} F_{n_{j}}\left(z ; d_{j}\right) \frac{d z}{z} \\
= & \left(d_{m+1} a, d_{m+1} / a ; q\right)_{n_{m+1}} \sum_{k=0}^{n_{m+1}} \frac{\left(q^{-n_{m+1}} ; q\right)_{k} q^{k}}{\left(q, d_{m+1} a, q^{1-n_{m+1}} a / d_{m+1} ; q\right)_{k}} \\
& \times \int_{C} \frac{\left(z^{2}, z^{-2} ; q\right)_{\infty}}{\left(q^{k} a z, q^{k} a / z, b z, b / z, c z, c / z, d z, d / z ; q\right)_{\infty}} \prod_{j=1}^{m} F_{n_{j}}\left(z ; d_{j}\right) \frac{d z}{z},
\end{aligned}
$$

where $m, n_{j}$ are nonnegative integer numbers, and $j=1,2, \cdots, m+1$.

We can derive the formula (7) from (6) and the following $q$-Saalschütz's summation (see Gasper \& Rahman, 2004, p. 17, Equation (1.7.2)):

$$
{ }_{3} \Phi_{2}\left(\begin{array}{ccc}
q^{-n}, & a, & f \\
& h, & a f q^{1-n} / h
\end{array} ; q, \quad q\right)=\frac{(h / a, h / f ; q)_{n}}{(h, h / a f ; q)_{n}} .
$$

Rewriting (8) as follows:

$$
\sum_{k=0}^{n} \frac{\left(q^{-n} ; q\right)_{k} q^{k}}{\left(q, h, q^{1-n} a f / h ; q\right)_{k}} \frac{1}{\left(q^{k} a, q^{k} f ; q\right)_{\infty}}=\frac{(h / a, h / f ; q)_{n}}{(h, h / a f ; q)_{n}} \frac{1}{(a, f ; q)_{\infty}} .
$$

In (9), replacing $(a, f)$ by $(a z, a / z)$ respectively, we get

$$
\sum_{k=0}^{n} \frac{\left(q^{-n} ; q\right)_{k} q^{k}}{\left(q, h, q^{1-n} a^{2} / h ; q\right)_{k}} \frac{1}{\left(q^{k} a z, q^{k} a / z ; q\right)_{\infty}}=\frac{1}{\left(h, h / a^{2} ; q\right)_{n}} \frac{F_{n}(z ; h / a)}{(a z, a / z ; q)_{\infty}} .
$$

Both sides of (10) multiply by

$$
\frac{\left(z^{2}, z^{-2} ; q\right)_{\infty}}{(b z, b / z, c z, c / z, d z, d / z ; q)_{\infty}} \prod_{j=1}^{m} F_{n_{j}}\left(z ; d_{j}\right) \frac{1}{z}
$$

then we find that

$$
\begin{aligned}
& \sum_{k=0}^{n} \frac{\left(q^{-n} ; q\right)_{k} q^{k}}{\left(q, h, q^{1-n} a^{2} / h ; q\right)_{k}} \frac{\left(z^{2}, z^{-2} ; q\right)_{\infty}}{\left(q^{k} a z, q^{k} a / z, b z, b / z, c z, c / z, d z, d / z ; q\right)_{\infty}} \frac{\prod_{j=1}^{m} F_{n_{j}}\left(z ; d_{j}\right)}{z} \\
= & \frac{1}{\left(h, h / a^{2} ; q\right)_{n}} \frac{\left(z^{2}, z^{-2} ; q\right)_{\infty}}{(a z, a / z, b z, b / z, c z, c / z, d z, d / z ; q)_{\infty}} \prod_{j=1}^{m} F_{n_{j}}\left(z ; d_{j}\right) \frac{F_{n}(z ; h / a)}{z} .
\end{aligned}
$$

Taking the contour integral on both sides of (12) with respect to variable $z$, denoting $n=n_{m+1}, h=d_{m+1} a$, we have the desired result.

Remark 1 The zero is single pole of the function $F_{n_{j}}\left(z ; d_{j}\right)(j=1,2, \cdots, m+1)$ in Unit Cirle. So we can use the similar method appeared in Askey and Wilson (1985) (or Costas-Santos \& Sánchez-Lara, 2013) to deform the Unit Circle. In the context of this paper, convergence of series is no issue at all because they are the terminating series.

\section{Some Applications}

Next, we will give some applications of (7). Since we assume the integrals are the same established condition as Theorem, we omit the condition in the following. 
Setting $m=0$ in (7), then employing (6), we get

Corollary 1 We have

$$
\begin{aligned}
& \frac{1}{2 \pi i} \int_{C} \frac{\left(z^{2}, z^{-2} ; q\right)_{\infty}}{(a z, a / z, b z, b / z, c z, c / z, d z, d / z ; q)_{\infty}} \frac{F_{n_{1}}\left(z ; d_{1}\right)}{z} d z \\
= & \frac{2\left(d_{1} a, d_{1} / a ; q\right)_{n_{1}}(a b c d ; q)_{\infty}}{(q, a b, a c, a d, b c, b d, c d ; q)_{\infty}} \sum_{k=0}^{n_{1}} \frac{\left(q^{-n_{1}}, a b, a c, a d ; q\right)_{k} q^{k}}{\left(q, d_{1} a, q^{1-n_{1}} a / d_{1}, a b c d ; q\right)_{k}} .
\end{aligned}
$$

Interchanging $a$ and $b$ in the above identity, we have

$$
\begin{aligned}
& \frac{1}{2 \pi i} \int_{C} \frac{\left(z^{2}, z^{-2} ; q\right)_{\infty}}{(a z, a / z, b z, b / z, c z, c / z, d z, d / z ; q)_{\infty}} \frac{F_{n_{1}}\left(z ; d_{1}\right)}{z} d z \\
= & \frac{2\left(d_{1} b, d_{1} / b ; q\right)_{n_{1}}(a b c d ; q)_{\infty}}{(q, a b, b c, b d, a c, a d, c d ; q)_{\infty}} \sum_{k=0}^{n_{1}} \frac{\left(q^{-n_{1}}, a b, b c, b d ; q\right)_{k} q^{k}}{\left(q, d_{1} b, q^{1-n_{1}} b / d_{1}, a b c d ; q\right)_{k}} .
\end{aligned}
$$

Combining (14) and (13), then replacing ( $\left.a b, a c, a d, d_{1} a, q^{1-n_{1}} a / d_{1}, a b c d\right)$ by $(a, d, c, d, e, f)$ respectively, giving the famous Sears' transformation of terminating balanced ${ }_{4} \Phi_{3}$ series:

Proposition 1 We have

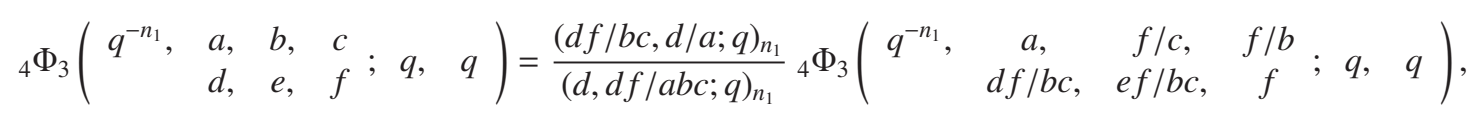

provided that def $=q^{1-n_{1}} a b c$.

Remark 2 Interchanging $d$ and $f$ in the above identity, noting that $d e f=q^{1-n_{1}} a b c$, we have $d f / b c=q^{1-n_{1}} a / e, d e / b c=$ $q^{1-n_{1}} a / f, d f / a b c=q^{1-n_{1}} / e$. If we substitute them into (15), we can conclude that Sears' transformation of terminating balanced ${ }_{4} \Phi_{3}$ series (see Gasper \& Rahman, 2004, p. 360, Equation III. 15) after some simplification:

$$
{ }_{4} \Phi_{3}\left(\begin{array}{cccc}
q^{-n_{1}}, & a, & b, & c \\
& d, & e, & f
\end{array} ; \quad q, \quad q\right)=\frac{(e / a, f / a ; q)_{n_{1}}}{(e, f ; q)_{n_{1}}} \Phi_{3}\left(\begin{array}{ccccc}
q^{-n_{1}}, & a, & d / c, & d / b \\
& d, & q^{1-n_{1}} a / e, & q^{1-n_{1}} a / f
\end{array} ; q, \quad q\right)
$$

where $\operatorname{def}=q^{1-n_{1}} a b c$.

Letting $m=1$ in (7), then applying (13), we have

Corollary 2 We have

$$
\begin{aligned}
& \frac{1}{2 \pi i} \int_{C} \frac{\left(z^{2}, z^{-2} ; q\right)_{\infty}}{(a z, a / z, b z, b / z, c z, c / z, d z, d / z ; q)_{\infty}} \frac{F_{n_{2}}\left(z ; d_{2}\right) F_{n_{1}}\left(z ; d_{1}\right)}{z} d z \\
= & \frac{2\left(d_{2} a, d_{2} / a ; q\right)_{n_{2}}(a b c d ; q)_{\infty}}{(q, a b, a c, a d, b c, b d, c d ; q)_{\infty}} \sum_{k=0}^{n_{2}} \frac{\left(q^{-n_{2}}, a b, a c, a d ; q\right)_{k} q^{k}\left(q^{k} d_{1} a, d_{1} / q^{k} a ; q\right)_{n_{1}}}{\left(q, d_{2} a, q^{1-n_{2}} a / d_{2}, a b c d ; q\right)_{k}} \\
& \times \sum_{k_{1}=0}^{n_{1}} \frac{\left(q^{-n_{1}}, q^{k} a b, q^{k} a c, q^{k} a d ; q\right)_{k_{1}} q^{k_{1}}}{\left(q, q^{k} d_{1} a, q^{1-n_{1}+k} a / d_{1}, q^{k} a b c d ; q\right)_{k_{1}}} .
\end{aligned}
$$

Interchanging $a$ with $b, n_{1}$ with $n_{2}, d_{1}$ with $d_{2}$ in the above identity, we have

$$
\begin{aligned}
& \frac{1}{2 \pi i} \int_{C} \frac{\left(z^{2}, z^{-2} ; q\right)_{\infty}}{(a z, a / z, b z, b / z, c z, c / z, d z, d / z ; q)_{\infty}} \frac{F_{n_{1}}\left(z ; d_{1}\right) F_{n_{2}}\left(z ; d_{2}\right)}{z} d z \\
= & \frac{2\left(d_{1} b, d_{1} / b ; q\right)_{n_{1}}(a b c d ; q)_{\infty}}{(q, a b, b c, b d, a c, a d, c d ; q)_{\infty}} \sum_{k=0}^{n_{1}} \frac{\left(q^{-n_{1}}, a b, b c, b d ; q\right)_{k} q^{k}\left(q^{k} d_{2} b, d_{2} / q^{k} b ; q\right)_{n_{2}}}{\left(q, d_{1} b, q^{1-n_{1}} b / d_{1}, a b c d ; q\right)_{k}} \\
& \times \sum_{k_{1}=0}^{n_{2}} \frac{\left(q^{-n_{2}}, q^{k} a b, q^{k} b c, q^{k} b d ; q\right)_{k_{1}} q^{k_{1}}}{\left(q, q^{k} d_{2} b, q^{1-n_{2}+k} b / d_{2}, q^{k} a b c d ; q\right)_{k_{1}}} .
\end{aligned}
$$

From (17) and (18), we obtain the following extension formula of Sears' transformations of terminating ${ }_{4} \Phi_{3}$ : 
Proposition 2 (An extension Sears' transformations of terminating ${ }_{4} \Phi_{3}$ ) We have

$$
\begin{aligned}
& \sum_{k=0}^{n_{2}} \frac{\left(q^{-n}, a b, a c, a d ; q\right)_{k} q^{k}\left(q^{k} d_{1} a, d_{1} / q^{k} a ; q\right)_{n_{1}}}{\left(q, d_{2} a, q^{1-n_{2}} a / d_{2}, a b c d ; q\right)_{k}} \\
& \times{ }_{4} \Phi_{3}\left(\begin{array}{ccccc}
q^{-n_{1}}, & q^{k} a b, & q^{k} a c, & q^{k} a d \\
& q^{k} d_{1} a, & q^{1-n_{1}+k} a / d_{1}, & q^{k} a b c d
\end{array} ; q, \quad q\right) \\
& =\frac{\left(d_{1} b, d_{1} / b ; q\right)_{n_{1}}}{\left(d_{2} a, d_{2} / a ; q\right)_{n_{2}}} \sum_{k=0}^{n_{1}} \frac{\left(q^{-n_{1}}, a b, b c, b d ; q\right)_{k} q^{k}\left(q^{k} d_{2} b, d_{2} / q^{k} b ; q\right)_{n_{2}}}{\left(q, d_{1} b, q^{1-n_{1}} b / d_{1}, a b c d ; q\right)_{k}} \\
& \times{ }_{4} \Phi_{3}\left(\begin{array}{ccccc}
q^{-n_{2}}, & q^{k} a b, & q^{k} b c, & q^{k} b d \\
& q^{k} d_{2} b, & q^{1-n_{2}+k} b / d_{2}, & q^{k} a b c d
\end{array} ; q, \quad q\right) .
\end{aligned}
$$

Remark 3 Setting $n_{2}=0$, we get (15) after some rearrangement and simplification.

Putting $m=2$ in (7), then applying (17), we find that

Corollary 3 We have

$$
\begin{aligned}
& \frac{1}{2 \pi i} \int_{C} \frac{\left(z^{2}, z^{-2} ; q\right)_{\infty}}{(a z, a / z, b z, b / z, c z, c / z, d z, d / z ; q)_{\infty}} \frac{F_{n_{3}}\left(z ; d_{3}\right) F_{n_{2}}\left(z ; d_{2}\right) F_{n_{1}}\left(z ; d_{1}\right)}{z} d z \\
= & \frac{2\left(d_{3} a, d_{3} / a ; q\right)_{n_{3}}(a b c d ; q)_{\infty}}{(q, a b, a c, a d, b c, b d, c d ; q)_{\infty}} \sum_{k=0}^{n_{3}} \frac{\left(q^{-n_{3}}, a b, a c, a d ; q\right)_{k} q^{k}\left(q^{k} d_{2} a, d_{2} / q^{k} a ; q\right)_{n_{2}}}{\left(q, d_{3} a, q^{1-n_{3}} a / d_{3}, a b c d ; q\right)_{k}} \\
& \times \sum_{k_{1}=0}^{n_{2}} \frac{\left(q^{-n_{2}}, q^{k} a b, q^{k} a c, q^{k} a d ; q\right)_{k_{1}} q^{k_{1}}\left(q^{k+k_{1}} d_{1} a, d_{1} / q^{k+k_{1}} a ; q\right)_{n_{1}}}{\left(q, q^{k} d_{2} a, q^{1-n_{2}+k} a / d_{2}, q^{k} a b c d ; q\right)_{k_{1}}} \\
& \times \sum_{k_{2}=0}^{n_{1}} \frac{\left(q^{-n_{1}}, q^{k+k_{1}} a b, q^{k+k_{1}} a c, q^{k+k_{1}} a d ; q\right)_{k_{2}} q^{k_{2}}}{\left(q, q^{k+k_{1}} d_{1} a, q^{1-n_{1}+k_{1}+k} a / d_{1}, q^{k+k_{1}} a b c d ; q\right)_{k_{2}}} .
\end{aligned}
$$

Similar as the process of getting Proposition 2.4, interchanging $a$ with $b, n_{1}$ with $n_{2}, d_{1}$ with $d_{2}$ in the above identity, we find that:

Proposition 3 (Another extension Sears' transformations of terminating ${ }_{4} \Phi_{3}$ ) We have

$$
\begin{aligned}
& \sum_{k=0}^{n_{3}} \frac{\left(q^{-n_{3}}, a b, a c, a d ; q\right)_{k} q^{k}\left(q^{k} d_{2} a, d_{2} / q^{k} a ; q\right)_{n_{2}}}{\left(q, d_{3} a, q^{1-n_{3}} a / d_{3}, a b c d ; q\right)_{k}} \\
& \times \sum_{k_{1}=0}^{n_{2}} \frac{\left(q^{-n_{2}}, q^{k} a b, q^{k} a c, q^{k} a d ; q\right)_{k_{1}} q^{k_{1}}\left(q^{k+k_{1}} d_{1} a, d_{1} / q^{k+k_{1}} a ; q\right)_{n_{1}}}{\left(q, q^{k} d_{2} a, q^{1-n_{2}+k} a / d_{2}, q^{k} a b c d ; q\right)_{k_{1}}} \\
& \times \sum_{k_{2}=0}^{n_{1}} \frac{\left(q^{-n_{1}}, q^{k+k_{1}} a b, q^{k+k_{1}} a c, q^{k+k_{1}} a d ; q\right)_{k_{2}} q^{k_{2}}}{\left(q, q^{k+k_{1}} d_{1} a, q^{1-n_{1}+k+k_{1}} a / d_{1}, q^{k+k_{1}} a b c d ; q\right)_{k_{2}}} \\
& =\frac{\left(d_{3} b, d_{3} / b\right)_{n_{3}}}{\left(d_{3} a, d_{3} / a\right)_{n_{3}}} \sum_{k=0}^{n_{3}} \frac{\left(q^{-n_{3}}, a b, b c, b d ; q\right)_{k} q^{k}\left(q^{k} d_{1} b, d_{1} / q^{k} b ; q\right)_{n_{1}}}{\left(q, d_{3} b, q^{1-n_{3}} b / d_{3}, a b c d ; q\right)_{k}} \\
& \times \sum_{k_{1}=0}^{n_{1}} \frac{\left(q^{-n_{1}}, q^{k} a b, q^{k} b c, q^{k} b d ; q\right)_{k_{1}} q^{k_{1}}\left(q^{k+k_{1}} d_{2} b, d_{2} / q^{k+k_{1}} b ; q\right)_{n_{2}}}{\left(q, q^{k} d_{1} b, q^{1-n_{2}+k} b / d_{1}, q^{k} a b c d ; q\right)_{k_{1}}} \\
& \times \sum_{k_{2}=0}^{n_{2}} \frac{\left(q^{-n_{2}}, q^{k+k_{1}} a b, q^{k+k_{1}} b c, q^{k+k_{1}} b d ; q\right)_{k_{2}} q^{k_{2}}}{\left(q, q^{k+k_{1}} d_{2} b, q^{1-n_{1}+k+k_{1}} b / d_{2}, q^{k+k_{1}} a b c d ; q\right)_{k_{2}}} .
\end{aligned}
$$

Remark 4 Letting $n_{3}=n_{2}=0$ in the above identity, we can get (15) again after some rearrangement and simplification. It is easy to see that interchanging $a$ with $c$, exchanging $n_{1}$ and $n_{3}, d_{1}$ and $d_{3}$, one can get other transformation formulas.

\section{Acknowledgements}

The author is supported by Jiangsu Overseas Research and Training Program for University Prominent Young and Middle-Aged Teachers and Presidents. The author are also supported by National Natural Science Foundation of 
China (No. 11371163) and National Natural Science Foundation of China (No. 11001098). The author thanks the referees and editors for their many valuable comments and suggestions.

\section{References}

Andrews, G. E., \& Askey, R. (1981). Another $q$-extension of the beta function. Proc. Amer. Math. Soc., 81(1981), 97-100. http://dx.doi.org/10.2307/2043995

Askey, R. (1981). A q-extension of Cauchy's form of the beta integral. Quart. J. Math. 32(3), $255-266$. http://dx.doi.org/10.1137/1027063

Askey, R., \& Wilson, J. (1985). Some basic hypergeometric polynomials that generalize Jacobi polynomials. Memoirs Amer. Math. Soc., 54(319) (Providence, Rhode Island, USA).

Costas-Santos, R. S., \& Sánchez-Lara, J. F. (2013). A survey on q-polynomials and their orthogonality properties. Retrieved from http://arxiv.org/pdf/1002.4657

Fang, J. -P. (2007). q-Differential operator identities and applications. J. Math. Anal. Appl., 332, 1393-1407. http://dx.doi.org/10.1016/j.jmaa.2006.10.087

Fang, J. -P. (2014). Note on a q-contour integral formula. Appl. Math. Comput. http://dx.doi.org/10.1016/j.amc.2014.01.155

Gasper, G., \& Rahman, M. (2004). Basic Hypergeometric Series (2nd ed.). Cambridge: Cambridge University Press.

Ismail, M. E. H., \& Masson, D. R. (1994). $q$-Hermite polynomials, biorthogonal rational functions, and $q$-beta integrals. Trans. Amer. Math. Soc., 346, 63-116. http://dx.doi.org/10.1090/S0002-9947-1994-1264148-6

Ismail, M. E. H., \& Masson, D. R. (1995). Some basic bilateral sums and integrals. Pacific J. Math., 170(2), 497-515. http://projecteuclid.org/euclid.pjm/1102370882

Jackson, F. H. (1910). On a q-definite integrals. Quart. J. Pure Appl. Math., 41, 193-203.

Liu, Z.-G. (2003). Some operator identities and $q$-series transformation formulas. Discrete Math., 265, $119-139$. http://dx.doi.org/10.1016/S0012-365X(02)00626-X

Wang, M.-J. (2010). A recurring $q$-integral formula. Appl. Math. Lett., 23, $256-260$. http://dx.doi.org/10.1016/j.aml.2009.10.004

\section{Copyrights}

Copyright for this article is retained by the author(s), with first publication rights granted to the journal.

This is an open-access article distributed under the terms and conditions of the Creative Commons Attribution license (http://creativecommons.org/licenses/by/3.0/). 